\title{
Analyzing the QoS Parameters in WiMAX Using Adaptive Modulation and Coding Schemes with HARQ
}

\author{
Komaldeep Kaur ${ }^{1}$ and Vinit Grewal ${ }^{2}$ \\ ${ }^{1}$ Student, M-Tech, ECE, GNDU RC Jalandhar \\ ${ }^{2}$ Assistant Professor, ECE Dept., GNDU RC Jalandhar \\ ${ }^{1}$ k.komal17118@gmail.com, ${ }^{2}$ vinitdhaliwal90@gmail.com
}

\begin{abstract}
In this paper, the performance of QoS parameters for video conferencing in WiMAX network has been analyzed using adaptive modulation and coding schemes (AMC) with Hybrid Automatic Repeat Request (HARQ). HARQ when used with AMC, proved to be most suitable for WiMAX because of its hybrid nature, as it takes both MAC and link layer into account. QoS performance parameters are evaluated using OPNET modeler 14.5.
\end{abstract}

Keywords: AMC, HARQ, QoS, WiMAX

\section{Introduction}

Worldwide Interoperability for Microwave Access is the emerging technology which promises to provide higher data rate up to 30 to $40 \mathrm{Mbps}$ and covers up to $50 \mathrm{~km}$. WiMAX supports both packet oriented transmission and standard mobile telephony over a large distance with its better performance in terms of throughput [10]. WiMAX is designed to support different types of traffic such as Voice-over-IP(VoIP), media streaming, online gaming, video conferencing, web browsing and instant messaging and many more. There are salient key features supported by WiMAX such as, QoS provides end to end IP based service, Flexible spectrum allocation with different channelization from 1.25 to $20 \mathrm{MHz}$, Security is enhanced with new authentication, Provision of high data rate with Multiple Input Multiple Output (MIMO) antenna techniques [1].

The WiMAX defines the physical layer (PHY) and Media Access Control (MAC) layer with the MAC consisting of several sublayers: MAC Convergence Sublayer (CS), MAC Common Part Sublayer (CPS) and MAC Security Sublayer[5].

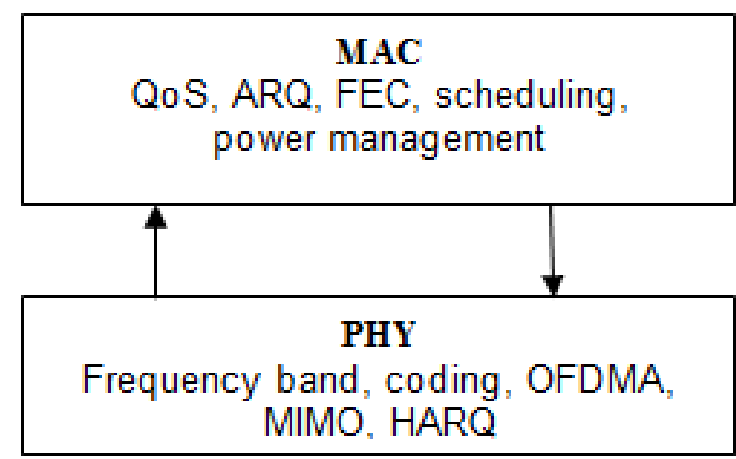

Figure 1. OSI Layer Model of WiMAX

PHY layer function is to process frames which are carried from upper layer to suitable form for transmission for wireless medium and all this process is done by mapping in OFDM, Forward Error Correction Coding (FCC), modulation and channel estimation. 
MAC layer is divided into several sublayers and its function is to transport packets from upper layer and arrange all the packets into MAC Protocol Data Unit (MPDU) which is then further passed to PHY layer. QoS is the most important parameter to determine the video service. It is the ability to control the parameters in network such as jitter, delay, throughput, bandwidth and packet loss in order to deliver network service. QoS ensure the network to perform at desired level. QoS means there should be low delay, low jitter, low loss, low latency and high throughput [7].

This paper is organized as follows. In Section 1, brief discussion about WiMAX, QoS and video application is given. Section 2 gives the background, introduces the adaptive modulation and coding scheme with HARQ. Modeling and simulation is presented in Section 3. Results and analysis is shown in Section 4 and Section 5 concludes the paper with future study.

\section{Background}

The author [9] concluded that using HARQ with AMC, radio resources usage had reduced and delay were also small. So both application delay and capacity have now been reduced. It has also been concluded that best way is to use AMC with HARQ. The author [1] shows that when AMC is used with HARQ low TCP delays are seen and the statistics also show that when ARQ is enabled in conjunction with HARQ, there is slight improvement in BLER but at the expense of very high UL frame usage. The work discuss about various issues for efficient QoS support and issues related to PHY and MAC layer. The author in [10] analyze that various WiMAX QoS parameters which are critical in determining the performance of a WiMAX network. An optimum WiMAX network should have a very low value of delay and packet dropped, whereas a very high value of throughput. The paper presents the analysis of QoS parameters and it was found that parameters that affect the QoS are jitter, throughput, delay and packet loss.

PHY layer of WiMAX use the adaptive modulation and coding (AMC) to match interference condition. In wireless communication systems requirement of data rate and spectral efficiency is increasing day by day therefore use of AMC has become a standard approach in wireless technologies such as WiMAX [1]. The idea to adapt the modulation and coding scheme is to achieve the highest spectral efficiency. Nodes lying in various regions use different modulation and coding schemes shown in Figure 2.

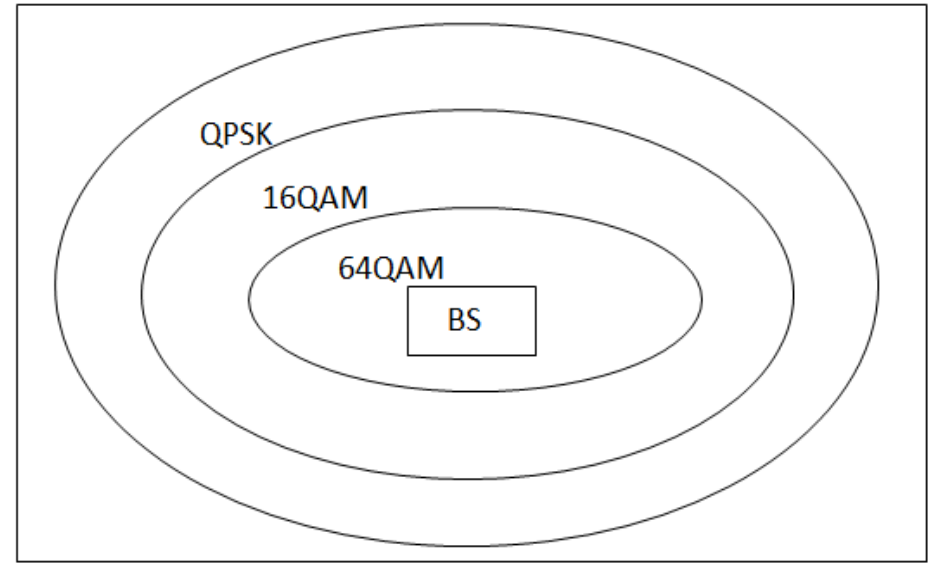

Figure 2. Adaptive Modulation and Coding

Automatic Repeat Request (ARQ) is a retransmission mechanism which operates at upper MAC layer and sometimes also referred as Radio Link Control (RLC). ARQ ensures in order delivery to the IP layer but MAC layer ARQ alone does not improve the spectral efficiency because retransmission cost of extra delay. Hybrid ARQ (HARQ) is 
another retransmission mechanism which operates at PHY layer, different from ARQ operating at MAC layer. HARQ allows the receiver to retransmit the packets but it performs soft combining of packets and therefore spectral efficiency also gets improved by $10-20 \%$.

\section{Modeling and Simulation}

One scenario has been developed for modeling and simulation. In this analysis of QoS for video conferencing is done over WiMAX network using 16QAM modulation scheme with HARQ. QoS for video conferencing in WiMAX is realized using OPNET Modeler 14.5 which is an extensive networking tool to analyze the performance of a network. The important parameters used in the simulation are summarized in Table 1.

Table 1. Simulation Parameters

\begin{tabular}{|l|l|}
\hline Parameters & Values \\
\hline PHY Layer & IEEE 802.16 \\
\hline BS transmission power & $0.5 \mathrm{~W}$ \\
\hline Base Station antenna gain & $15 \mathrm{dBi}$ \\
\hline Mobile Station antenna gain & $-1 \mathrm{dBi}$ \\
\hline Modulation schemes & $16-$ QAM3/4 \\
\hline Pathloss parameter & Vehicular environment \\
\hline Radius of cell & $2 \mathrm{~km}$ \\
\hline $\begin{array}{l}\text { Number of fixed and mobile } \\
\text { nodes }\end{array}$ & 28 mobile, 20fixed \\
\hline PHY profile & Wireless OFDMA 20 MHz \\
\hline Simulation time(sec) & 480 \\
\hline Efficiency mode & $\begin{array}{l}\text { Mobility and Ranging } \\
\text { enabled }\end{array}$ \\
\hline Node position & Random \\
\hline
\end{tabular}

In simulation in order to have fairer comparison, rtPS (real time polling service) has been used for video conferencing in WiMAX. In this scenario 4 hexagonal cells are taken and each cell has one Base Station. The BS connected to the IP backbone via ppp_adv link. The node 0 and vod_server is connected to backbone through ppp_sonet_oct1 duplex link. 


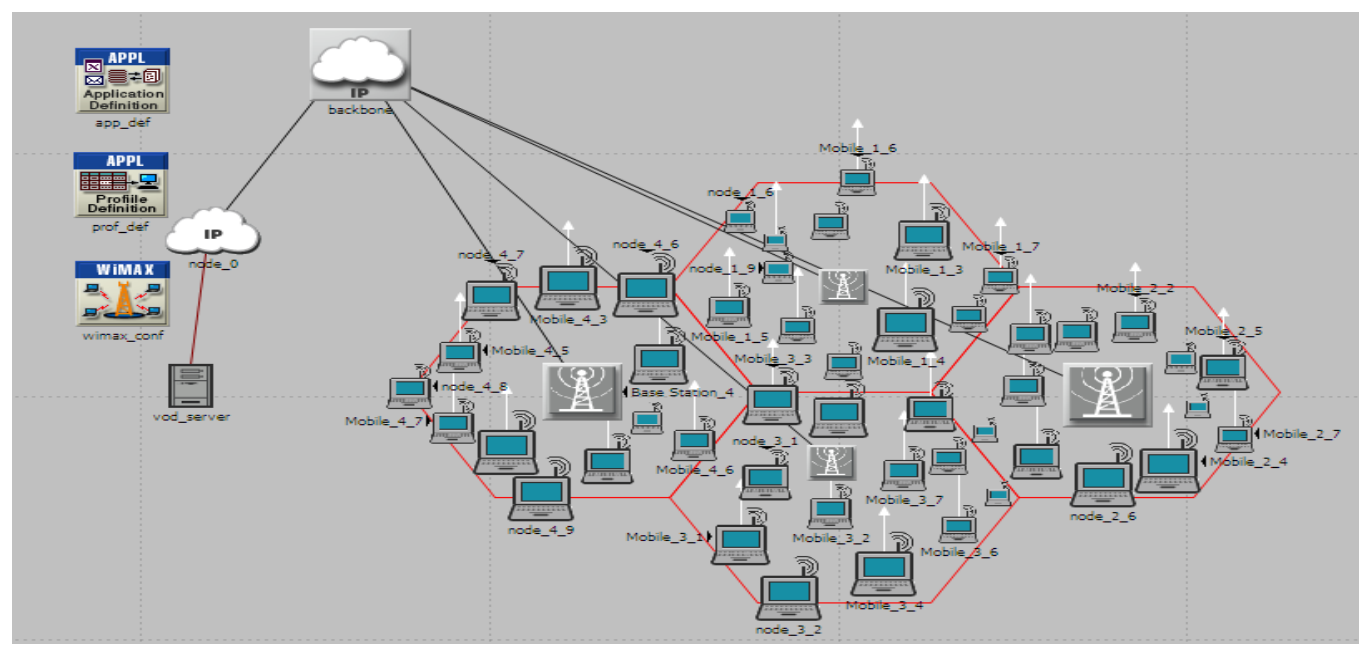

Figure 3. Video Conferencing Over WiMAX Network

\section{Results and Analysis}

The performance analysis of 16QAM and 16QAM using HARQ have been done by measuring several QoS parameters such as throughput, delay and load.

\subsection{Throughput}

Throughput is the average data rate of successful message delivery over a communication channel. Throughput should always be high for successful delivery. The result in the Figure 4 indicates that when HARQ scheme is used then the throughput of the network increased. Hence the efficiency of the network also improved by $20 \%$.

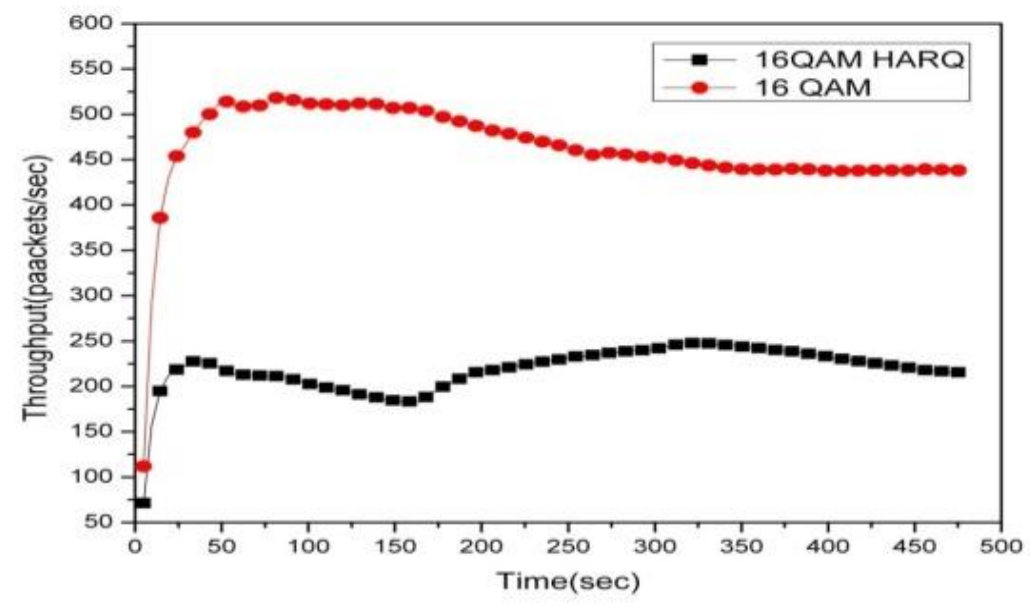

Figure 4. Throughput

\subsection{Load}

Load represents the total load submitted to WiMAX layers by all higher layers. Load rate is less when HARQ scheme is used with AMC and it further improves the performance of the network. Figure 5 represents the result of load. 


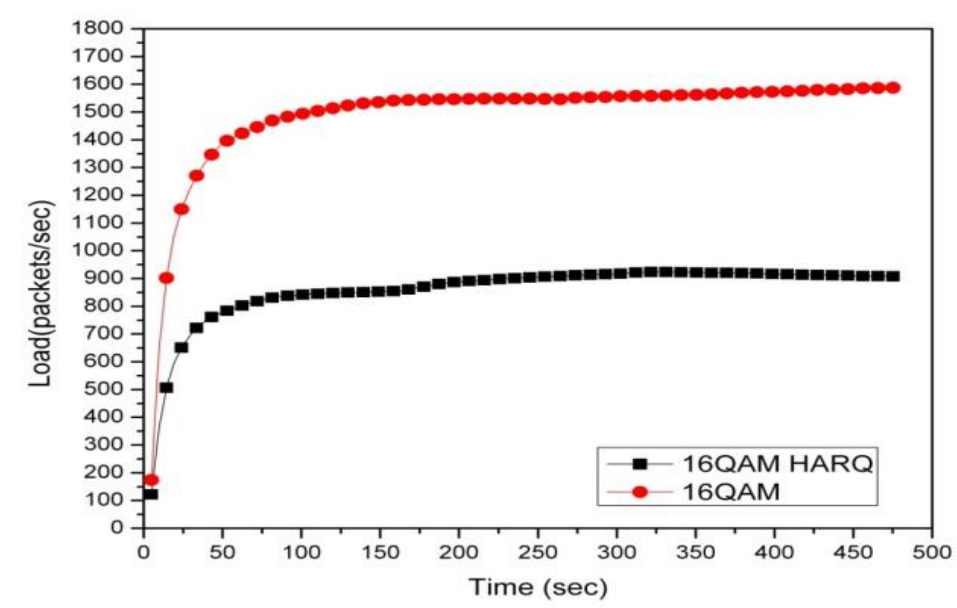

Figure 5. Load

\subsection{Delay}

Delay represents the time taken by packet to be transferred from source to destination. From the values obtained it can be inferred that delay is less when hybrid scheme is used.

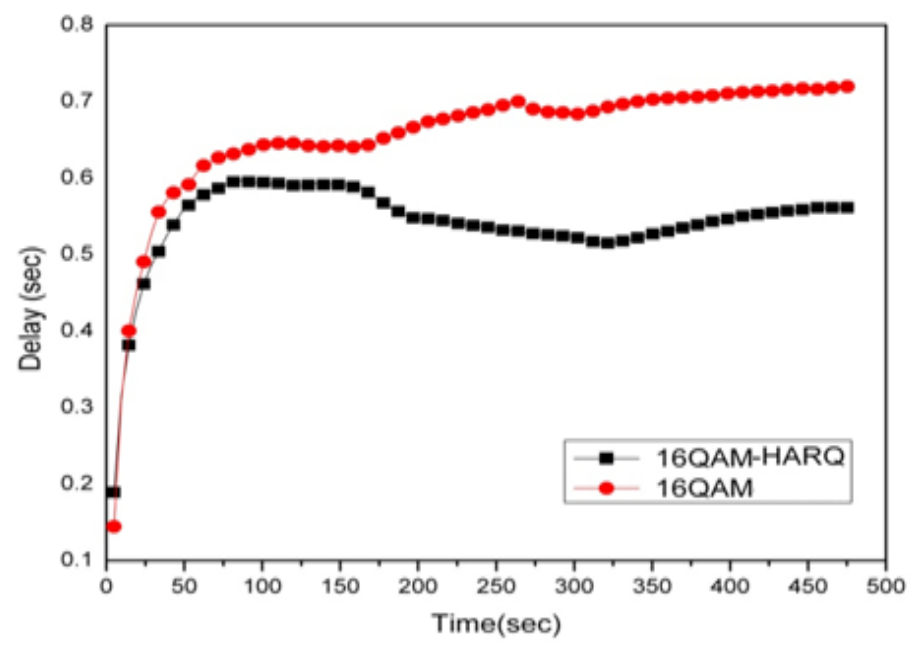

Figure 6. Delay

Comparative study of QoS parameters of video conferencing in WiMAX network shows that when AMC is used with HARQ scheme spectral efficiency of the networks gets increased by $10-20 \%$. 
Table 2. Qos Performance Using HARQ Scheme with 16QAM Modulation Scheme

\begin{tabular}{|l|l|l|}
\hline QoS Parameter & 16QAM3/4 (average) & 16QAM3/4 using HARQ (average) \\
\hline Throughput & 219.0387 & 464.0675 \\
\hline Load & 1492.201 & 858.9133 \\
\hline Delay & 0.542232 & 0.396859 \\
\hline
\end{tabular}

The average values of all the QoS parameters are enlisted in Table 2, it is observed that Adaptive modulation and coding schemes with HARQ performs the best. Thus results clearly indicate that performance of QoS parameters improved when HARQ scheme is used for video conferencing in WiMAX network.

\section{Conclusion}

The performance of various QoS mechanisms for both the fixed and mobile environments in WiMAX network is realized. The investigation is also carried out for AMC scheme with HARQ and its effect on the QoS performance of WiMAX network is studied. The performance has been evaluated in terms of delay, load and throughput and the efficiency of network is improved by $10-20 \%$. It has been concluded that best strategy is to use AMC with the HARQ. In future, analysis should be done for other modulation schemes and QoS performance needs to be improved further to achieve higher throughput and low delay.

\section{References}

[1] A. Q. Ansari, "WiMAX Network Optimization -Analyzing Effects of Adaptive Modulation and Coding Schemes Used in Conjunction with ARQ and HARQ", Seventh Annual Communications Networks and Service Research Conference, (2009).

[2] P. Grover and M. Chawla, "Performance Analysis of QoS For WiMAX Using OPNET", IEEE International Conference on Computer, Communication and Control (IC4-2015).

[3] B. Kaarthick and V. J. Yeshwenth, "Performance analysis of Video Conferencing and Multimedia application Service over WiMAX”, 2009 IEEE International Advance Computing Conference(IACC 2009)Patiala, India, (2009).

[4] M. A. Gumaa and K. H. Bilal, "Performance Evaluation of QoS Parameters in WiMAX Network", International Journal of Science and Research (IJSR), vol. 3, no. 9, (2014).

[5] J. L. Vizcaíno, "WiMAX", IEEE 802.16, (2008).

[6] M. Patider and R. dubey, "Performance Analysis of WiMAX 802.16e Physical Layer Model," IEEE, (2012).

[7] K. H. Teo, T. Zhifeng and J. Zhang, "The Mobile Broadband WiMAX Standard Standards in a Nutshell”, IEEE Signal Processing Magazine, vol. 24, (2007), pp. 144-148.

[8] J. Benes and J. Prokopec, "Efficiency of Encoding Schemes in Mobile WiMAX", IEEE Journal and Transactions, (2011), pp. 1-4.

[9] I. Adhicandra, "Using AMC and HARQ to Optimize System Capacity and Application Delays in WiMAX Networks", Journal of Telecommunications, vol. 2, no. 2, (2010).

[10] J. G. Andrews, "Fundamentals of WiMAX: Understanding Broadband Wireless Networking", (Prentice Hall Communications Engineering and Emerging Technologies Series) Prentice Hall, (2007). 


\section{Authors}

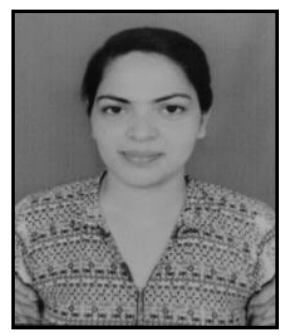

Komaldeep Kaur, she is pursuing M-Tech in Electronics and Communication from GNDU, RC, Jalandhar. Her research interests include wireless communication and computer networks.

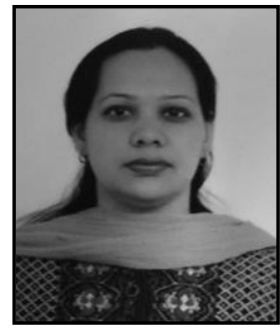

Vinit Grewal, she received the $\mathrm{PhD}$ degree in Electronics and Communication from National Institute of Technology, Jalandhar in WiMax networks in 2013. Her research interests include digital communication, signal processing and wireless networking. 
International Journal of Multimedia and Ubiquitous Engineering Vol.12, No.8 (2017) 